\title{
Dialisi peritoneale: dialisi marginale? La sinergia è senza dubbio da preferire rispetto alla contrapposizione
}

\author{
Alberto Santoboni \\ U.O.C. Nefrologia e Dialisi, Ospedale "L. Parodi Delfino", Colleferro (RM)
}

\begin{abstract}
Peritoneal dialysis: marginal dialysis?
Abstract. In my routine clinical practice I often asked myself why two complementary dialysis techniques (hemodialysis and peritoneal dialysis) should be in conflict.

Possible explanations may refer to the competence in performing peritoneal dialysis, the dominant position of private practice hemodialysis units, and the shortage of nurses and physicians.

Nevertheless we must provide complete information to our patients approaching renal replacement therapy, as to offer them the most comfortable dialysis conditions to alleviate the disease's discomfort.
\end{abstract}

Key words: Peritoneal dialysis, Hemodialysis, Ongoing treatment

Conflict of interest: None.

Financial support: None.

Accettato: 2 Settembre 2013

Fin dai primi anni della mia specializzazione in ambito nefrologico, la contrapposizione tra peritonealisti e anti-peritonealisti riempiva parte delle giornate passate in reparto. I vantaggi dell'una e dell'altra metodica venivano passati al setaccio con dovizia di particolari.

Spesso le dispute si risolvevano senza vinti né vincitori; sembrava di assistere alle storiche dispute tra i sostenitori di Mazzola e quelli di Rivera oppure tra i sostenitori di Motta o di Gimondi.

Nella mia attività clinica degli anni successivi, mi sono spesso chiesto perché due metodiche che reputo complementari debbano escludersi, sì, proprio escludersi, perché, altrimenti, non ci sarebbero regioni con un'alta incidenza di ricorso al trattamento intracorporeo e altre con una bassissima incidenza $(2 \%)$ e non ci sarebbero U.O.C. con il $50 \%$ di pazienti in trattamento peritoneale mentre, in altre U.O.C., non vengono inseriti pazienti in dialisi con questa metodica.

Quale sia la ragione di tale disparità di atteggiamento si può solamente ipotizzare; si può immaginare che la conoscenza e la competenza in ambito peritonealistico possano giocare un ruolo, anche se personalmente non credo che professionisti con anni di esperienza non abbiano colmato eventuali lacune di informazione ereditate da scuole di specializzazione poco attente.
La maggiore incidenza di strutture private può giustificare il minore ricorso alla metodica ma non la totale assenza di tali prestazioni che, peraltro, non possono essere erogate in ambito privatistico; si assiste, invece, alla totale assenza di trattamenti peritoneali in ambito pubblico.

Neanche la carenza di personale può essere chiamata in causa, in quanto è proprio il risparmio di personale uno dei punti di forza del trattamento, al pari della valutazione dei costi, che depongono a favore della dialisi peritoneale, aspetto che le nostre amministrazioni non dovrebbero ignorare.

Da questa disamina molto parziale, traggo solo alcune personali valutazioni: in termini percentuali, la metodica peritoneale non potrà avere maggiore penetranza, soprattutto in alcune regioni virtuose, dove amministratori avveduti hanno saputo dare input favorevoli. In altre regioni, invece, dove la scarsa lungimiranza degli amministratori ha impedito un corretto ed equilibrato ricorso ai trattamenti peritoneali, noi operatori del settore dovremmo fare uno sforzo culturale e umano per garantire ai nostri pazienti la possibilità di una convivenza con la malattia nel modo più accettabile possibile, dando loro la possibilità di decidere, quando possibile, la strategia migliore per limitare al massimo lo stravolgimento della loro vita quotidiana e la gestione del lavoro e degli affetti. 
Credo sia il volere di tutti quelli che, come me, non parteggiano né per Mazzola né per Rivera e che, offrendo tutte le opzioni possibili ai nostri pazienti, forniamo un servizio completo, sia dal punto di vista professionale che da quello umano, in quanto evitiamo di negare ai nostri pazienti quelle opzioni terapeutiche che possono, ne sono convinto, migliorare, e non di poco, la qualità della loro convivenza con la malattia e, quindi, con la propria esistenza.

\section{Riassunto}

Nella mia attività clinica mi sono spesso chiesto perché due metodiche (emodialisi e dialisi peritoneale), che reputo complementari, debbano escludersi. Quale sia la ragione di tale disparità di atteggiamento si può solamente ipotizzare: la competenza in ambito peritonealistico, la maggiore incidenza di strutture private e la carenza di personale.

Quale che sia la ragione, noi operatori abbiamo il dovere di fornire un servizio completo, sia dal punto di vista professionale che da quello umano, in quanto, così facendo, garantiremo ai nostri pazienti le migliori condizioni possibili per affrontare il peso della malattia.
Parole chiave: Dialisi peritoneale, Emodialisi, Trattamento complementare

Dichiarazione di conflitto di interessi: L'Autore dichiara di non avere conflitto di interessi.

Contributi economici degli autori: L'Autore dichiara di non aver ricevuto sponsorizzazioni economiche per la preparazione dell'articolo.

Indirizzo degli Autori:

Dr. Alberto Santoboni

U.O.C. Nefrologia e Dialisi

Ospedale "L. Parodi - Delfino"

Piazza A. Moro, 1

00034 Colleferro (Roma)

alberto.santoboni@aslromag.it 